\title{
La plataforma e-revist@s del portal Tecnociencia. Una experiencia basada en open access
}

\author{
Por Elena Fernández Sánchez, Luis Rodríguez Yunta y Juan Francisco Heras
}

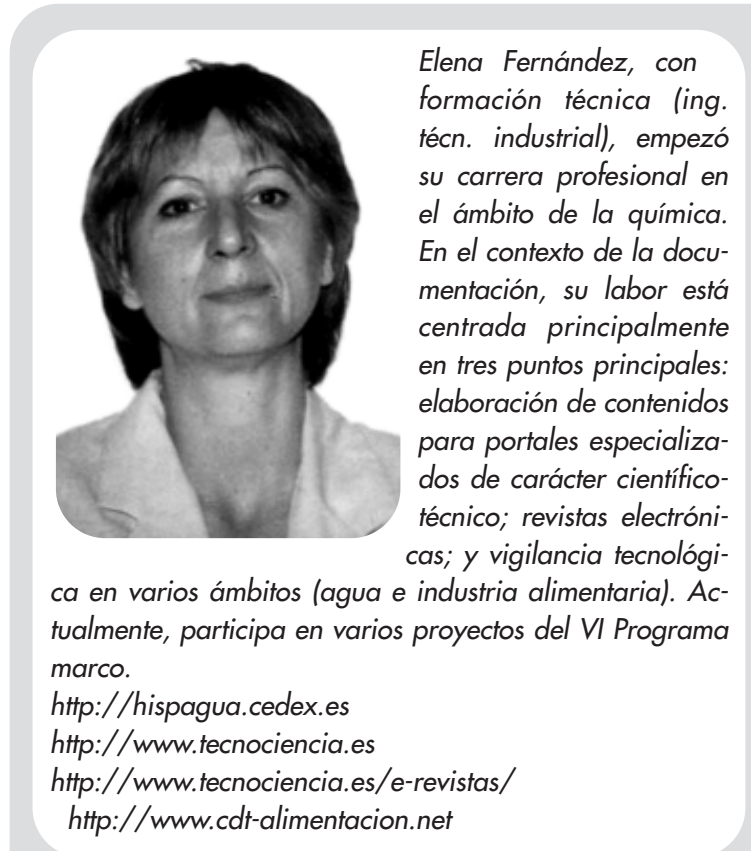

Resumen: Se analiza el caso de la plataforma e-revist@s como una iniciativa dentro del marco del movimiento open access. En esta experiencia se describen los objetivos del proyecto, requisitos de las revistas seleccionadas, contenidos actuales de la plataforma y las diferentes relaciones del proyecto con los editores de las revistas.

\begin{abstract}
Luis Rodríguez Yunta trabaja desde 1986 en el Cindoc en el mantenimiento de la base de datos ISOC (en las áreas de historia, arqueología, antropología y estudios latinoamericanistas). Ha participado desde su inicio en el proyecto del portal Tecnociencia, así como en otros proyectos de elaboración de publicaciones bibliográficas, estudios sobre la producción científica española, impartición de cursos y colaboración en la red europea Redial. Desde 2000 es vocal de la asociación profesional Sedic, de la que actualmente es secretario general.
\end{abstract}
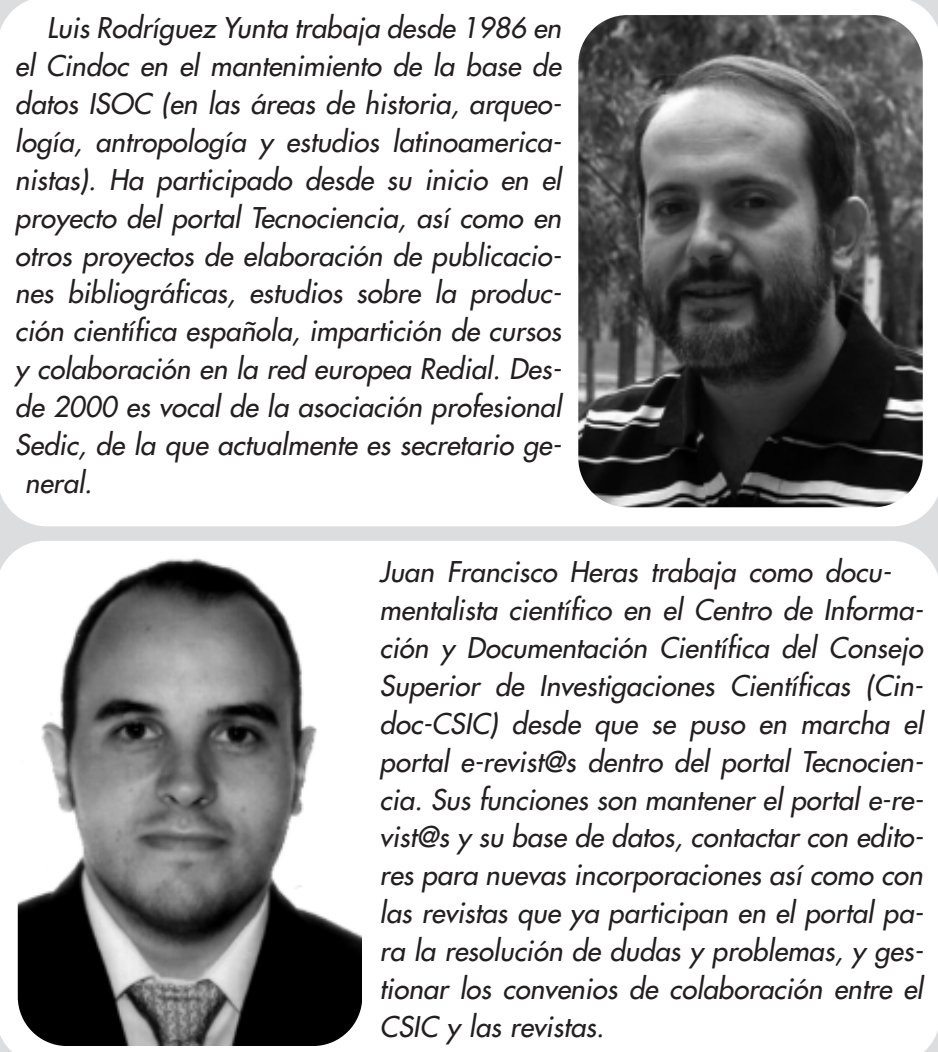

Juan Francisco Heras trabaja como documentalista científico en el Centro de Información y Documentación Científica del Consejo Superior de Investigaciones Científicas (Cindoc-CSIC) desde que se puso en marcha el portal e-revist@s dentro del portal Tecnociencia. Sus funciones son mantener el portal e-revist@s y su base de datos, contactar con editores para nuevas incorporaciones así como con las revistas que ya participan en el portal para la resolución de dudas y problemas, y gestionar los convenios de colaboración entre el CSIC y las revistas.

Palabras clave: Sistemas de información, Bases de datos documentales, Revistas científicas electrónicas, Internet, Acceso abierto.

Title: The e-revist@s platform of the Tecnociencia portal. An experience based on the open access initiative

Abstract: The aim of this paper is to describe the objectives, requirements and contents of the e-revist@s platform, as well as the relations between the project and the different journal publishers.

Keywords: Information systems, Documental databases, Scientific e-journals, Internet, Open access.

\section{E-REVIST@S ES UNA INI-} CIATIVA QUE PRETENDE promover tanto la presencia en internet como la visibilidad de las revistas científicas publicadas en España y Latinoamérica que hayan pasado por unos determinados filtros de calidad (véase anexo I).

El proyecto ha desarrollado un portal especializado en información sobre las revistas científicas y la edición electrónica, y presenta además una serie de recursos seleccionados, ofreciendo información adicional para todos los interesados en este campo.

http://www.tecnociencia.es/ e-revistas/

\section{Descripción}

El punto de partida se apoyó en los debates mantenidos en el marco de una lista de distribución creada en RedIris en mayo de 2003 en la que participaron documentalistas y editores de revistas científicas. Las conclusiones de este foro sirvieron como caldo de cultivo para la propuesta lanzada a los editores por el
Centro de Información y Documentación Científica (Cindoc) y la Fundación Española para la Ciencia y la Tecnología (Fecyt).

La plataforma digital está alojada en el portal Tecnociencia y está abierta a la participación de las revistas científicas electrónicas españolas y latinoamericanas ya existentes o de nueva creación que cumplan unos requisitos de calidad rigurosos basados en un sistema de evaluación normalizado y validado internacionalmente. El acceso a ellas ha de ser gratuito, sin que ello 


\section{MultiSearch \\ Metabúsqueda... ¡realmente simple y rápicla!}

Desarrollado por

CSA ILLUMINA

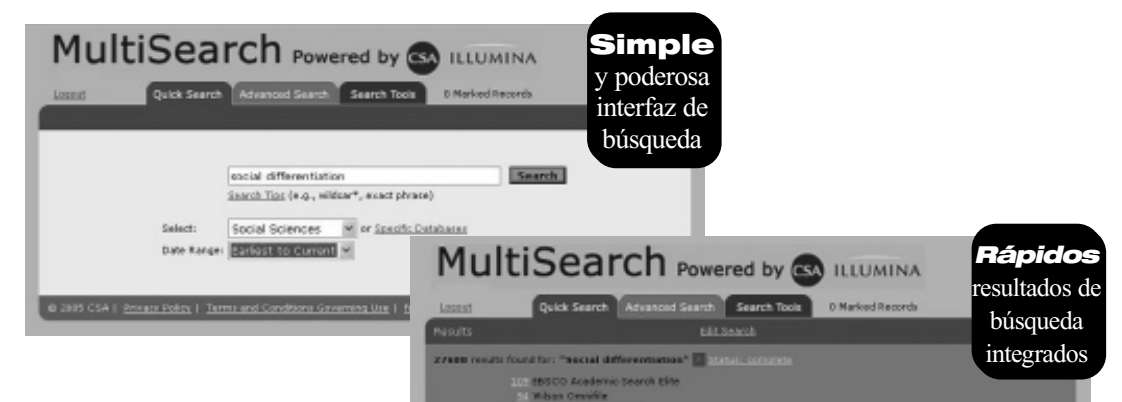

Con MultiSearch, ahorre tiempo, esfuerzo y dinero...

- Solución de bajo costo y bajo riesgo para metabúsquedas

- Búsquedas simultáneas en más de 2.000 fuentes

- Enlaces directos a la fuente para realizar más búsquedas, visualizaciones y exploraciones

La forma más simple y más rápida de realizar búsquedas en más de 2.000 recursos electrónicos

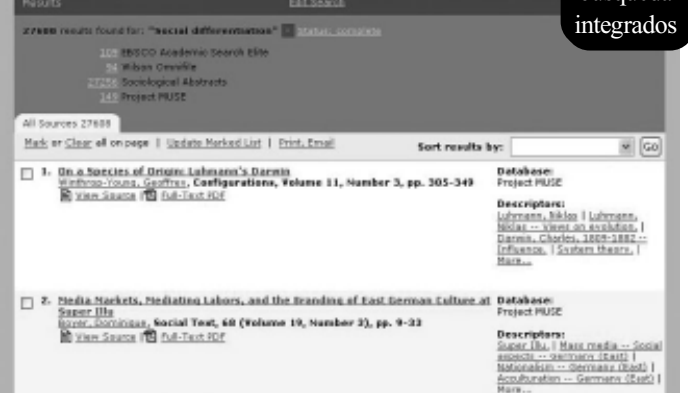

Implementación
simple y rápida

MultiSearch ofrece una solución simple y rápicla para realizar metabúsquedas que requieran una implementación mínima. MultiSearch ya es compatible con casi todos los principales recursos electrónicos, lo que la convierte en una solución eficiente y fácil de implementar.

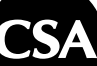

7200 Wisconsin Avenue Suite 601 Bethesda MD 20814 USA $\mathbf{t}+1301.961 .6700$ f +1301.961 .6720$

www.csa.com
Si desea obtener más información acerca de la interfaz de MultiSearch, póngase en contacto con CSA (multisearch@csa.com) menoscabe los principios de calidad que deben regir los textos de las publicaciones integradas en el proyecto. Dicho acceso se realiza a través de la interrogación de una base de datos o mediante navegación a través de su directorio. Tal y como está diseñada la plataforma permitirá, además de consultar las publicaciones, crear un repositorio de todas aquellas que deseen formar parte del portal.

En el proyecto participan varios agentes:

-Financiación: Fecyt, fundación dependiente del Ministerio de Educación y Ciencia.

-Gestión documental y coordinación: Cindoc,

-Coordinación del software: Centro Técnico de Informática del Csic (Cti).

-Grupo de trabajo: Cindoc, Cti, Instituto de Agroquímica $y$
Tecnología de Alimentos del Csic (Iata), Departamento de Biblioteconomía y Documentación de la Universidad Complutense de $\mathrm{Ma}$ drid y Fecyt.

-Editores de las revistas: todos aquellos que deseen formar parte del proyecto (véase anexo II).

\section{Arquitectura lógica general}

Está basada en UPS (universal preprint service) y en su derivación propuesta por la iniciativa OAI (open archives initiative), descrita por Barrueco y Subirats $(2003)^{1}$. Básicamente, OAI-PMH utiliza transacciones http para emitir preguntas y obtener respuestas entre un servidor o archivo y un cliente o servicio recolector de metadatos. El segundo puede pedir al primero que le envíe metadatos según determinados criterios. En respuesta, el servidor devuelve un conjunto de registros en formato $\mathrm{xml}$, incluyendo identificadores (una url por ejemplo) de los objetos descritos en cada registro.

El sistema identifica dos entidades lógicas: el proveedor de metadatos (data provider) y el de servicios (service provider). El primero de ellos, que en nuestro caso sería la revista electrónica, es el responsable de la publicación y almacenamiento de los recursos en un repositorio y del mantenimiento de sus metadatos para que puedan "recolectarse" desde los proveedores de servicios. El proveedor de servicios, por su parte, se encarga de recopilar los datos para proporcionar servicios de valor añadido en función de los metadatos que ha recogido. Entre los tipos de ayudas que el proveedor de servicios puede proporcionar, está la creación de una interfaz de búsqueda unificada para todos los repositorios. 


\begin{tabular}{|c|c|}
\hline \multicolumn{2}{|c|}{ Características básicas } \\
\hline 1. & Mención del cuerpo editorial (consejo editorial) \\
\hline 2. & Contenido científico (al menos el 40\%) \\
\hline 3. & $\begin{array}{l}\text { Antigüedad mínima de un año (las de nueva creación estarán condicionadas a } \\
\text { superar ese periodo) }\end{array}$ \\
\hline 4. & Identificación de los autores \\
\hline 5. & Identificación de la identidad editora \\
\hline 6. & Mención del director \\
\hline 7. & Url \\
\hline \multicolumn{2}{|c|}{ Características de presentación de la revista } \\
\hline 8. & Navegación y funcionalidad \\
\hline 9. & Mención de la periodicidad \\
\hline 10. & Tabla de contenidos (índice o sumario) \\
\hline 11. & Membrete bibliográfico al inicio del artículo \\
\hline 12. & Afiliación de los autores (lugar de trabajo) \\
\hline 13. & Recepción y aceptación de originales \\
\hline \multicolumn{2}{|c|}{ Características de gestión y política editorial } \\
\hline 14. & ISSN \\
\hline 15. & Definición de la revista \\
\hline 16. & Sistema de selección de originales \\
\hline 17. & Evaluadores externos \\
\hline 18. & Autores externos (apertura institucional de la autoría de los trabajos) \\
\hline 19. & Apertura editorial \\
\hline 20. & Servicios de información \\
\hline 21. & Cumplimiento de la periodicidad \\
\hline \multicolumn{2}{|c|}{ Características de los contenidos } \\
\hline 22. & Instrucción a los autores \\
\hline 23. & Elaboración de las referencias bibliográficas \\
\hline 24. & Exigencia de originalidad \\
\hline 25. & Título en el idioma propio y en inglés \\
\hline 26. & Resumen en el idioma propio y en inglés \\
\hline 27. & Palabras clave en el idioma propio y en inglés \\
\hline 28. & Metaetiquetas (Dublin Core) \\
\hline 29. & Buscadores \\
\hline 30. & Servicios de valor añadido \\
\hline
\end{tabular}

Anexo I. Criterios de calidad editorial definidos por el sistema Latindex para revistas electrónicas y aplicados en la plataforma e-revist@s http://www.latindex.org

\section{Relación entre los editores y e-revist@s}

En función de la infraestructura informática y las posibilidades del editor,e-revist@s contempla tres opciones para la participación de éstos:

-Que el editor publique la revista electrónica y posea su propio proveedor de datos. En este caso, Tecnociencia se limita a capturar los metadatos del editor mediante el protocolo OAI-PMH e incorporar un enlace al texto completo disponible en la web del editor.

-Que el editor tenga la publicación en su propio servidor, pero no posea un proveedor de datos.
En este caso, los metadatos deben grabarse en la plataforma de $T e c$ nociencia por métodos convencionales y actuar como proveedor de datos sobre dicha revista para cualquier otro sistema de información que utilice el protocolo $O A I-P M H$.

-Que el editor no disponga de la versión electrónica y no posea 
un gestor de revistas ni un proveedor de datos propios y quiera alojar la revista en el servidor de Tecnociencia. La plataforma actuará aquí como socio tecnológico para la edición digital de la publicación.

En los dos primeros casos, $\mathrm{Tec}$ nociencia ofrece opcionalmente la posibilidad de actuar como repositorio de un mirror de la publicación. Por tanto, se trata de un sistema descentralizado, que puede dar acceso a artículos disponibles en las sedes web de los editores o bien a las copias accesibles en su propio archivo digital.

- Revista internacional de medicina y ciencias de la actividad fisica y del deporte. http://cdeporte.rediris.es/revista/revista.html

- RED. Revista de educación a distancia. http://www.um.es/ead/red/

-Tiempos modernos.

http://www.tiemposmodernos.org/

—Relieve. Revista electrónica de investigación y evaluación educativa.

http://www.uv.es/RELIEVE/

-ACE. Revista de enología.

http://www.acenologia.com/

-Percepnet. Ciencias sensoriales y de la percep-

ción.

http://www.acenologia.com/

-Ecotropía.

http://www.ecotropia.com/

- Geofocus. Revista internacional de ciencia y tecnología de la información geográfica. http://geofocus.rediris.es/

-Panace@. Boletín de medicina y traducción. http://www.medtrad.org/panacea.html

—Inteligencia artificial.

http://aepia.dsic.upv.es/revista/revista.html

-Hispania nova. Revista de historia contemporánea.

http://hispanianova.rediris.es/

- Scripta Nova. Revista electrónica de geografia y ciencias sociales.

http://www.ub.es/geocrit/noval.htm

-Biblio 3W. Revista bibliográfica de geografia y ciencias sociales.

http://www.ub.es/geocrit/bw-ig.htm

—EliEs. Estudios de lingüística del español. http://elies.rediris.es/

- Revista de procesamiento del lenguaje natural. http://www.sep/n.org/revistaSEPLN/revisSEPLN.htm

- BiD, textos universitaris de biblioteconomia $i$ documentació.

http://www.ub.es/biblio/bid/

-Anales de psicología.

http://www.um.es/analesps/

-Archivos de zootecnia.

http://www.uco.es/organiza/servicios/publica/az/az. $h t m$

\section{Contenidos y servicios de e-revist@s}

Desde el 1 de enero de 2004, fecha de inicio del proyecto, los resultados obtenidos en abril de 2005 son los siguientes:

$-\mathrm{N}^{\mathrm{o}}$ de revistas que se han adherido al proyecto: 56 .

$-\mathrm{N}^{\mathrm{o}}$ de artículos introducidos:

\subsection{8 .}

Los editores se adhieren al proyecto a través de un convenio de colaboración entre el Cindoc y la revista en cuestión. El objetivo del

-Athenea. Revista de pensamiento e investigación social.

http://antalya.uab.es/atheneal

- Anales de documentación: revista de biblioteconomía y documentación.

http://www.um.es/fccd/anales/

-Boletín de la Asociación de Geógrafos Españoles (AGE).

http://www.ieg.csic.es/age/boletin.htm

- Gazeta de antropología.

http://www.ugr.es/ pwlac/Welcome.html

- Contributions to science.

http://www.cat-science.com/

-Revista psicológica.

http://www.uv.es/psicological

—Revista investigaciones geográficas.

http://www.cervantesvirtual.com/portal/IIGG/

— Revista española de salud pública.

http://scielo.isciii.es/scielo.php?script=sci_serial\&pi $d=1 / 35-5727 \& / n g=e s \& n r m=i s o$

-Investigaciones económicas.

http://www.funep.es/invecon/ie_entrada.asp

-Enfermería global.

http://www.um.es/egloball

-Revista de toxicología.

http://tox.umh.es/aetox/index.htm

-Revista electrónica interuniversitaria de formación del profesorado.

http://www.aufop.org/publica/reifp/index.asp

-Revista pasado y memoria.

http://www.ua.es/hum.contemporaneas/ pasado-memoria

-The Spanish journal of psycology. http://www.ucm.es/sjp

-Ámbitos. Revista andaluza de comunicación http://www.ull.es/publicaciones/latina/ambitos/ ambitos.htm

—Boletín de pediatría

http://www.sccalp.org/boletin.htm

- Seguimiento farmacoterapéutico. http://www.cipf-es.org/esp/sft.htm

-Revista aquatic.

http://www.revistaaquatic.com/

- Revista ars pharmaceutica.

http://www.ugr.es/ ars/ars.htm equipo de trabajo es seguir difundiendo el proyecto y contactar con nuevas revistas que puedan sumarse a la plataforma.

En las figuras 1 y 2 se muestra la distribución de revistas y artículos por temática respectivamente.

\section{Servicios añadidos}

El servidor de e-revist@s proporciona un sistema de búsqueda avanzado, posibilita la navegación por índices de artículos, ofrece información de las características de las revistas y una serie de recursos

-Revista geologica-acta.

http://www.geologica-acta.com

-Anales del Sistema Sanitario de Navarra. http://www.cfnavarra.es/salud/anales/default.html

— La revista agricultura técnica.

http://www.scielo.cl/scielo.php?script=sci_serial\&p $d=0365-2807 \& \operatorname{lng}=$ es

-Revista acimed.

http://scielo.sld.cu/scielo.php?script=sci_serial\&pid

$=1024-9435 \& \operatorname{lng}=$ es\& $\mathrm{nrm}=$ iso

-Revista puzzle.

http://www.revista-puzzle.com

- Revista canarias pediátrica

http://www.comtf.es/pediatria/canarias_ pediátrica.htm

—Revista paleopatología.

http://www.ucm.es/info/aep/paleopatologia.htm

- Revista española de documentación científica. http://www.cindoc.csic.es/redc/redc.html

- Revista EURE.

http://www.scielo.cl/scielo.php?script=sci_serial\&pi $d=0250-7 / 6 /$ \&lng=es\&nrm=iso

-Apuntes de ciencia y tecnología http://www.cica.es/aliens/aacte/

— LinRed. Revista lingüística en la red. http://www.linred.com/

- Redvet. Revista electrónica de veterinaria. http://www.veterinaria.org/revistas/redvet

—Revista de investigación clínica y farmacéutica. http://www.revistainvestigacion.pfizer.es/

- Revista ra ximhai.

http://www.uaim.edu.mx/webraximhai/ra_ ximhai.htm

- Revista panamericana de salud pública. http://publications.paho.org/spanish/descriptions.cfm

— Revista ecosistemas.

http://www.aeet.org/ecosistemas/revista.htm

-Convergencia.

http://convergencia.uaemex.mx

- Signa. Revista de la Asociación Española de Semiótica.

http://www.cervantesvirtual.com/hemerotecal signalindex.shtml

- Revista de procesos y métricas de sistemas.

http://www.aemes.fi.upm.es/rpm/rpm.php

Anexo II. Lista de revistas presentes en la plataforma e-revist@s en abril de 2005 
relacionados con su edición electrónica.

Finalmente, durante el año 2005 uno de los principales objetivos es ofrecer a los editores el número de accesos por artículo y revista.

\section{Comparación con otros proyectos iberoamericanos}

En el ámbito iberoamericano se han desarrollado, con anterioridad o de forma paralela, otros sistemas que comparten objetivos similares a los de la plataforma $e$-revist@s. En muchos casos se trata de proyectos complementarios que pueden colaborar entre sí. Todas estas iniciativas contribuyen a aumentar la visibilidad de las revistas electrónicas españolas y latinoamericanas en condiciones de libre acceso, aunque sus planteamientos particulares difieren de los de Tecnociencia:

-SCiELO: hemeroteca digital cooperativa para la publicación electrónica de revistas científicas en internet que cumplan unos requisitos estrictos de calidad. Ha sido desarrollado por la Fundación de Apoyo a la Investigación del Estado de São Paulo (Fapesp) y el Centro Latinoamericano y del Caribe de Información en Ciencias de la Salud (Bireme) en colaboración con una red iberoamericana de instituciones relacionadas con la comunicación científica y editores científicos. Es un sistema multidisciplinar, aunque en el caso español se ha limitado a las publicaciones de ciencias de la salud con la participación de un total de 21 títulos. Se caracteriza por establecer un modelo normalizado de edición para todas las revistas que se suman al proyecto. Por ello, parece dirigido más a títulos que deseen iniciar un proyecto de edición electrónica que a la difusión de publicaciones que ya están consolidadas con su propio formato. http://www.scielo.org http://www.fapesp.br http://www.bireme.br

- RedALyC: portal de acceso a revistas científicas iberoamericanas de ciencias sociales y humanidades de libre acceso. Es un proyecto impulsado por académicos de la Facultad de Ciencias Politicas y Administración Pública de la Universidad Autónoma del Estado de México. A esta iniciativa se han sumado 23 publicaciones españolas. Se trata de un proyecto limitado a disciplinas humanísticas y sociales.

http://www.redalyc.org

-Temaria: plataforma de consulta de artículos de revistas electrónicas de biblioteconomía y documentación. Ha sido desarrollado por un grupo de investigación de la Facultad de Biblioteconomía y Documentación de la Universidad de Barcelona. Actualmente incluye 5 títulos y se trata de un sistema limitado a una única disciplina.

http://bidoc.ub.es/temaria/

—Dialnet: servicio de alerta y base de datos bibliográfica multidisciplinar especializada en revistas hispanas desarrollado por la biblioteca de la Universidad de La Rioja en un entorno de cooperación bibliotecaria, principalmente de las universidades del G-9. Actúa como servidor $O A I$ para alojar la versión electrónica de revistas que deseen digitalizarse (unas 15 actualmente). Sin embargo, no se trata propiamente de una plataforma para revistas electrónicas, sino de un servicio añadido ligado a un producto bibliográfico referencial de carácter general. http://dialnet.unirioja.es

—Revicien: portal de acceso a revistas científicas españolas, diseñado por la empresa Ciencia Digital y financiado por el Ministerio de Educación y Ciencia. Aunque permite acceder a algunas publicaciones a texto completo en acceso libre, se trata de una iniciativa que no se limita a revistas electrónicas y que no incluye publicaciones de ciencias sociales ni humanidades. http://www.revicien.net

Por todo lo expuesto, la plataforma e-revist@s de Tecnociencia constituye un sistema de información diferenciado de las restantes iniciativas por varios aspectos:

—su planteamiento es completamente multidisciplinar (frente a RedALyC, Temaria, Revicien o SciELO España),

— está basado sólo en publicaciones electrónicas de libre acceso (frente a Dialnet o Revicien),

- es flexible en el establecimiento de diferentes niveles de relación con los editores, aunando el

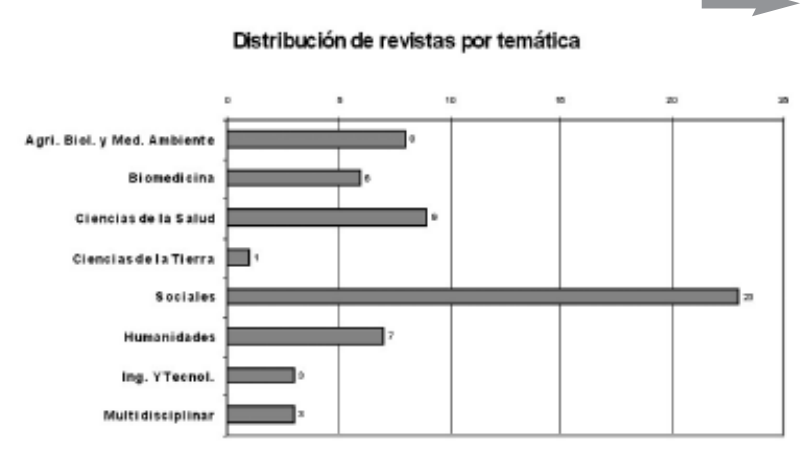

Figura 1

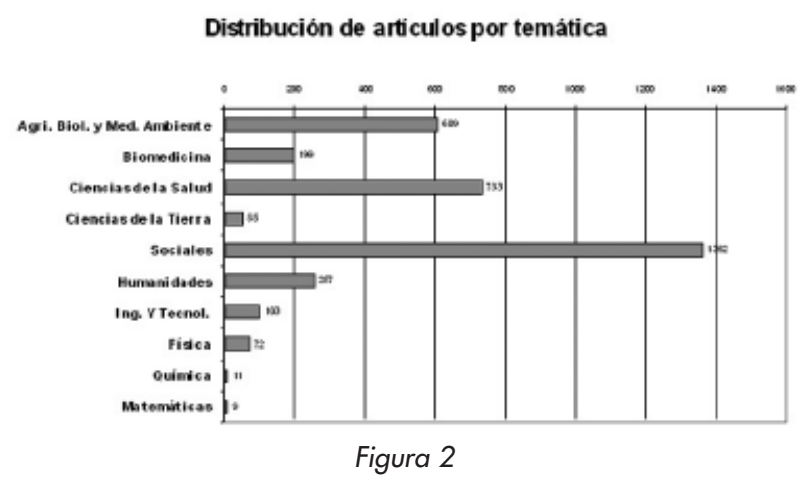




\section{doc6 en 30 segundos}

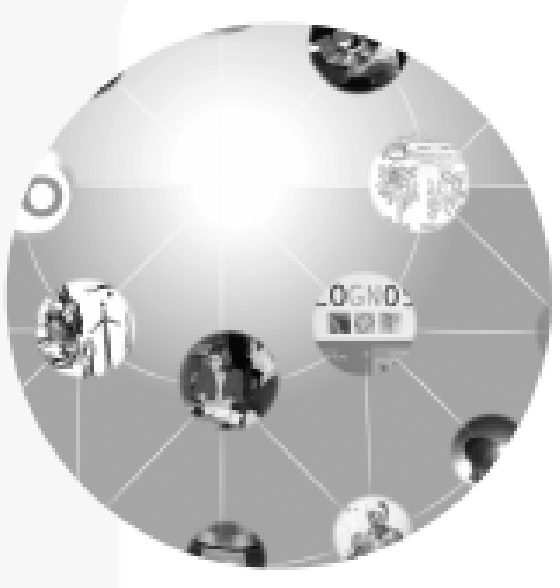

La empresa

Creada en el año 1988, especializada en gestión de la información y la documentación.

Certificada con la ISO 9001:2000 por Bureau Veritas desde Septiembre del 2000 en las siguientes actividades:

a CONSULTORES EN RECURSOS DE INFORMACION

GESTIÓN DEL CONOCIMIENTO

INTRANET/EXTRANET/INTERNET

EDICIÓN ELECTRÓNICA

I PROVEEDORES DE RECURSOS ELECTRÓNICOS DE INFORMACIÓN - SOLUCIONES EN GESTIÓN DOCUMENTAL

\section{CURSOS DE FORMACIÓN}

\section{Nuestros valores}

Innovación permanente.

Satisfacción del cliente. Calidad

Profesionalidad

Cooperación con el cliente

Confidencialidad

Responsabilidad social y valores éticos

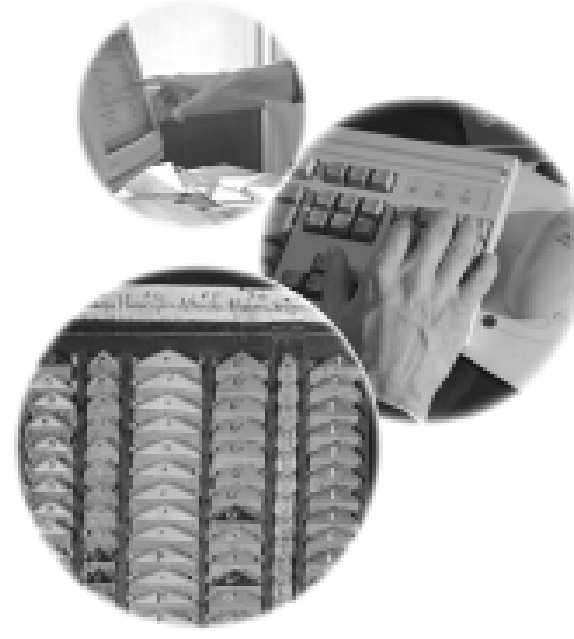

Nuestra misión

\section{Contribuir}

a aumentar

la eficiencia

de empresas

y organizaciones

a través de una

eficaz gestión

de la información

y el conocimiento.

\section{Los clientes}

El principal activo de la empresa, junto con sus empleados.

Más de 900 clientes: empresas, laboratorios, administraciones públicas, universidades, hospitales, bufetes de abogados, museos, fundaciones, colegios profesionales.

\section{Nuestros profesionales}

Una eficaz suma de conocimientos: informáticos y documentalistas con experiencia

El $75 \%$ son licenciados superiores o diplomados en informática o documentación. 
aporte descentralizado de metadatos con la opción de alojamiento de las publicaciones que lo deseen, y

- está insertado en un portal de divulgación científica con una oferta variada de contenidos.

\section{Conclusiones}

La plataformae-revist@s es un proyecto aún en fase de consolidación que pretende contribuir al posicionamiento en internet de las revistas científicas electrónicas publicadas en España y Latinoamérica. En estos países de habla hispana es necesario desarrollar herramientas para luchar contra el fenómeno conocido como la "ciencia perdida", asegurando la visibilidad y el acceso universal a la literatura científica editada en publicaciones nacionales.

En este contexto es también imprescindible establecer criterios de control de calidad que den garantías a los usuarios y aumenten el impacto social y científico de estos documentos. En esta misma línea están trabajando otros proyectos iberoamericanos que pueden considerarse complementarios al de Tecnociencia.

La edición electrónica posibilita nuevas vías de comunicación científica y agiliza la transmisión de datos y conocimientos. El protocolo $O A I-P M H$ facilita la construcción de sistemas de información que se alimenten de forma automática y descentralizada. Gracias a ello, los editores de revistas científicas pueden mantener su independencia de diseño y participar en diferentes plataformas de consulta, entre las cuales Tecnociencia responde a un modelo multidisciplinar.
E-revist@s se caracteriza además por tratarse de un portal de divulgación científica que ofrece recursos relacionados con las revistas científicas, el mundo editorial y el movimiento a favor del acceso abierto a las publicaciones. Su contenido se dirige tanto a la comunidad científica y académica como al sector empresarial y a la sociedad en general.

\section{Nota}

\footnotetext{
1. Barrueco, José Manuel; Subirats, Imma. "Open archives initiative. Protocol for metadata harvesting (OAI-PMH): descripción, funciones y aplicación de un protocolo". En: El profesional de la información, 2003, v. 12, n. 2, pp. 99-106.
}

Elena Fernández Sánchez; Luis Rodríguez Yunta; Juan Francisco Heras. Csic, Cindoc, Madrid.

elenaf@cindoc.csic.es

luisry@cindoc.csic.es

maga@cindoc.csic.es 